\title{
Tratamiento quirúrgico de las cardiopatías congénitas: Resultados de 213 procedimientos consecutivos
}

DIEGO ABDALA ${ }^{1}$, GLADYS LEJBUSIEWICZ ${ }^{2}$, GUILLERMO POSE ${ }^{3}$, GUILLERMO TOUYÁ ${ }^{4}$, JUAN RIVA ${ }^{5}$, LUIS LIGÜERA ${ }^{4}$, MAURICIO PASTORINO $^{6}$, SILVIA PÉREZ ${ }^{7}$, SERRANA ANTÚNEZ ${ }^{8}$, DANTE PICARELLI ${ }^{9}$

1. Pediatra. Postgrado Cardiología Pediátrica.

2. Ex Profesora Adjunta de Anestesia.

3. Pediatra Neonatólogo. Postgrado Cardiología Pediátrica.

4. Cirujano. Residente de Cirugía Cardíaca.

5. Profesor Adjunto de Anestesia.

6. Asistente de Anestesia.

7. Médico Perfusionista. Cardiopatías Congénitas

8. Cardióloga Pediatra. Jefe Hemodinamia. Depto. Cardiología. Cardiopatías Congénitas.

9. Cirujano Cardíaco. Jefe Depto de Cirugía Cardíaca. Cardiopatías Congénitas. Departamento de Cardiopatías Congénitas. Centro Cardiológico Americano.

\begin{abstract}
Surgical treatment of congenital heart diseases: results of 213 procedures

Background: Important improvements in surgical treatment of congenital heart diseases and increasing number of corrective surgeries over palliation. Objective: Evaluate the surgical results of 213 procedures performed in a center of our country. Method: Retrospective study that includes medical and surgical records from the database of the Cardiological American Center, enrolling 213 consecutive cardiac surgery procedures for congenital heart disease during 39 months (January 2003 - March 2006). The analysis focused on morbidity and mortality at hospital discharge, comparing the results with previous data published. Results: $87.8 \%$ were corrective surgical procedures. Mortality rate and complications at hospital discharge were $7 \%$ and $16 \%$, respectively. Conclusions: Mortality rate found is similar to most of the published series. There is a great tendency towards corrective surgical procedures. The highest mortality rate occured in the newborn group with complex cardiac malformations.

(Key words: Heart defects, congenital surgery, cardiac surgical procedures).

Rev Arch Pediatr Urug 2006; 77(3): 237-243
\end{abstract}

Fecha recibido: 23 de junio de 2006.

Fecha aprobado: 2 de agosto de 2006.

\section{Correspondencia:}

Dante Picarelli

Isabelino Bosch 2469. Montevideo, Uruguay.

E-mail: picarelli54@hotmail.com 


\section{RESUMEN}

Dado el progreso del tratamiento quirúrgico de las cardiopatías congénitas, y el predominio de las cirugías correctivas con respecto a las paliativas, hemos querido evaluar los resultados quirúrgicos, en forma individual y comparativa, de 213 procedimientos quirúrgicos por cardiopatía congénita realizados en un único centro de nuestro país. Sujetos y Métodos: Se revisaron retrospectivamente los registros médicos y quirúrgicos de la base de datos del Centro Cardiológico Americano, identificándose 213 cirugías cardíacas consecutivas por cardiopatía congénita en un período de 39 meses (desde enero de 2003 hasta marzo de 2006). Se analizó la morbimortalidad al alta, comparándose con la literatura internacional. Resultados: La mortalidad al alta fue de 7\% y el porcentaje de complicaciones de $16 \%, 87,8 \%$ de los procedimientos fueron correctivos. Conclusiones: La mortalidad total al alta se encuentra dentro de los límites referidos en muchos de los estudios publicados. Hay una marcada tendencia en favorecer las cirugías correctivas. La mayor mortalidad la posee el grupo de pacientes recién nacidos con cardiopatías complejas.

(Palabras clave: cardiopatías congénitas, cirugía, procedimientos quirúrgicos cardíacos).

Rev Arch Pediatr Urug 2006; 77(3): 237-243

\section{Introducción}

El tratamiento quirúrgico de las cardiopatías congénitas no ha cesado de progresar en los últimos 50 años ${ }^{1,2}$. Desde el primer cierre de una comunicación interventricular empleando circulación extracorpórea (CEC), en 1954³ , muchos han sido los avances obtenidos en las distintas áreas de la especialidad.

Las técnicas quirúrgicas, de anestesia, CEC $\mathrm{y}$ cuidados postoperatorios han adquirido tal sofisticación y desarrollo que constituyen una verdadera subespecialidad dentro de la cirugía cardíaca.

El éxito final de un procedimiento quirúrgico no depende solamente de un gesto técnicamente bien ejecutado, sino del ensamblaje perfecto de cada uno de los engranajes que participan en las distintas etapas del tratamiento.

Por su parte, la bioingeniería ha venido desarrollando materiales cada vez más adaptados a pacientes más pequeños, con superficies más biocompatibles minimizando la reacción inflamatoria provocada por éstos y permitiendo corregir cardiopatías congénitas complejas con CEC en recién nacidos (RN) y prematuros de muy bajo peso ${ }^{4}$.

El viejo concepto de que un paciente portador de una cardiopatía congénita tenía pocas oportunidades de sobrevivir hasta la edad adulta, hoy se ha revertido ${ }^{3}$.

Una de las razones fundamentales ha sido la corrección temprana de las alteraciones hemo- dinámicas provocadas por las anomalías estructurales, lo cual ha permitido evitar las deletéreas consecuencias fisiopatológicas sobre el miocardio y los demás parénquimas ${ }^{1}$. Por las razones antes expuestas, las cirugías correctivas con CEC han adquirido tal confiabilidad que aún en RN de bajo peso se prefieren a las paliativas, que quedaron reservadas para casos muy precisos debido a la mayor morbimortalidad determinada por la sumatoria de la ocasionada por cada cirugía y la no menos despreciable acontecida durante el intervalo transcurrido entre las mismas ${ }^{1,5-10}$.

Con el fin de evaluar los resultados del tratamiento quirúrgico por cardiopatías congénitas, hemos analizado retrospectivamente en forma individual y comparativa los resultados de 213 procedimientos quirúrgicos consecutivos por cardiopatía congénita realizados en un único centro de nuestro país.

\section{Sujetos y Método}

Se efectuó una revisión retrospectiva de los registros médicos y quirúrgicos de la base de datos del Centro Cardiológico Americano (Sanatorio Americano), que permitió identificar 213 procedimientos quirúrgicos consecutivos por cardiopatía congénita durante un período de 39 meses (enero de 2003 a marzo de 2006). Los mismos representaron $15 \%$ del total de actividad quirúrgica de dicho centro. 
El 96\% de las cirugías fueron efectuadas por el mismo cirujano. El manejo perioperatorio no varió a lo largo del período.

La circulación extracorpórea se realizó a una temperatura que varió entre $24^{\circ} \mathrm{C}$ y $30^{\circ} \mathrm{C}$, manteniendo un $\mathrm{Ht} \%$ mayor a $25 \%$, una saturación venosa de oxígeno mayor a $75 \%$ y una lactacidemia menor a $5 \mathrm{mos} / \mathrm{l}$. Con el fin de disminuir la concentración de factores proinflamatorios y aumentar el Ht\% se realizó hemofiltración convencional durante la CEC salvo en los pacientes con más de $10 \mathrm{Kg}$, en los que se efectuó cierre de comunicación interventricular (CIV) o comunicación interauricular (CIA).

En los pacientes con peso menor a $10 \mathrm{~kg}$ se exigió para la salida de CEC un $\mathrm{Ht} \%$ entre 42\%-45\%. Se utilizó en todos los casos cardioplejia blanca extracelular, repetida invariablemente cada 20 min luego de la dosis de inducción. Los shunt sistémico-pulmonares fueron realizados por esternotomía mediana, y los banding de la arteria pulmonar por toracotomía ántero lateral izquierda. El protocolo de fármacos inotrópicos incluyó el uso casi exclusivo de milrinona y adrenalina. Dependiendo del ritmo diurético postcirculación extracorpórea (menor o igual a $2,5 \mathrm{ml} / \mathrm{kg} /$ hora en las primeras 36 horas) se administró furosemide en goteo continuo u horario, asociado según necesidad a hidroclorotriazida y espironolactona.

En los RN y lactantes; con el fin de evitar la hemodilución y la hipooncosis, que aumentaría la extravasación al tercer espacio provocada por el aumento de la permeabilidad capilar (síndrome inflamatorio post CEC); se utilizó, para la reposición de volumen, exclusivamente concentrado globular o albúmina al 20\% en función de $\mathrm{Ht} \%$. El plasma fresco se reservó para los trastornos de la crasis con sangrado clínico (protocolo del Servicio de Cirugía Cardíaca del Hospital Necker Enfants Malades, París).

Para evaluar los resultados, se definió mortalidad como la muerte acontecida antes del alta hospitalaria. El mismo criterio se utilizó para las complicaciones.

\section{Resultados}

El 64,8\% (n=138) fueron cirugías con CEC. El promedio de edades fue de 5,7 años (1 día-
63 años) siendo 68\% menores de 12 meses (38,6\% recién nacidos). Según los criterios de complejidad (tabla 1), las cardiopatías fueron agrupadas en simples (48\%), medianamente complejas (29,5\%) y complejas (22,5\%).

El 87,8\% $(\mathrm{n}=187)$ de los procedimientos quirúrgicos fueron correctivos, 9,8\% $(\mathrm{n}=21)$ paliativos [banding de arteria pulmonar $(n=6)$, shunt sistémico-pulmonar $(\mathrm{n}=13)$, atrioseptostomía $(\mathrm{n}=2)$ ] y el $2,4 \%$ restante miscelánea de procedimientos entre los que se incluyen colocación de marcapaso, extracción de trombo, plicatura diafragmática, etc.

La mortalidad intraoperatoria fue de 1,9\% $(n=4)$; con los restantes 11 decesos acontecidos antes del alta configuran una mortalidad total de 7\% $(n=15) .73 \%(n=11)$ de los fallecidos eran portadores de cardiopatías complejas, y $27 \%(n=4)$ restante de mediana complejidad.

No hubo decesos en el grupo con cardiopatías simples. Salvo un solo paciente de 6 meses de edad, no falleció ningún paciente mayor de 3 meses [53,3\% $(n=8)$ fueron recién nacidos y $40 \%(n=6)$ pertenecían al grupo de 31 días a 3 meses].

Ocurrieron 34 complicaciones en 213 procedimientos quirúrgicos (16\%) (tabla 2), 15 (44\%) resultaron fatales.

Las causas de mortalidad se encuentran en la tabla 2.

Los cuatro decesos intraoperatorios fueron cardiopatías complejas: una atresia tricúspide con vasos normopuestos y foramen oval restrictivo que requirió atrioseptostomía quirúrgica, dos procedimientos de Norwood estadio I por ventrículo izquierdo hipoplásico (uno de los cuales, según el estudio anatomopatológico, asoció estenosis de venas pulmonares) y una corrección de truncus arterioso tipo II que presentó falla cardíaca intraoperatoria luego de una reposición masiva por severo trastorno de la crasis.

Siete pacientes fallecidos en el postoperatorio también eran portadores de cardiopatías complejas: nacimiento anómalo de rama pulmonar derecha de la aorta ascendente que presentó una crisis severa de hipertensión arterial pulmonar refractaria al tratamiento. Tres pacientes con ventrículos únicos que asociaban en un 
Tabla 1. Tipo de cardiopatías

\begin{tabular}{|c|c|}
\hline Simples & $n=96$ \\
\hline Ductus arterioso permeable & 23 \\
\hline $\mathrm{CIA}$ & 28 \\
\hline CIV & 26 \\
\hline Anillo vascular & 3 \\
\hline $\mathrm{CIA}+\mathrm{RVPAP}$ & 4 \\
\hline RVPAP & 2 \\
\hline Membrana subaórtica & 10 \\
\hline Mediana complejidad & $\mathbf{n}=\mathbf{5 9}$ \\
\hline Coartación aorta & 15 \\
\hline Tetralogía de Fallot & 21 \\
\hline Coartación de aorta + CIV & 2 \\
\hline CAVC & 9 \\
\hline CAVT & 1 \\
\hline CAVP & 1 \\
\hline Estenosis tracto salida ventrículo derecho & 1 \\
\hline Aneurisma seno Valsalva + EMV + CIV & 1 \\
\hline Endocarditis bacteriana activa & 1 \\
\hline $\mathrm{EMV}+\mathrm{CIV}$ & 1 \\
\hline $\mathrm{IAO}$ & 2 \\
\hline Mega auricula derecha & 1 \\
\hline CIV + estenosis subaórtica & 1 \\
\hline Insuficiencia mitral & 2 \\
\hline Complejas & $n=45$ \\
\hline VU & 6 \\
\hline TGA & 6 \\
\hline TGA + coartación aorta & 1 \\
\hline RVPAT supra & 3 \\
\hline RVPAT intra & 1 \\
\hline RVPAT infra + coartación aorta + VU & 1 \\
\hline Rama pulmonar aorta ascendente & 1 \\
\hline Triada de Shone & 1 \\
\hline DTSVD + atresia pulmonar & 3 \\
\hline DTSVD & 2 \\
\hline Atresia pulmonar a septo íntegro & 1 \\
\hline Estenosis supravalvular aórtica & 3 \\
\hline Aneurisma aorta ascendente & 1 \\
\hline CAVC + coartación de aorta & 2 \\
\hline $\mathrm{EAO}+$ coartación de aorta + CIV & 1 \\
\hline Truncus & 2 \\
\hline Ventrículo izquierdo hipoplásico & 2 \\
\hline Valvulopatía aórtica & 1 \\
\hline Tetralogía de Fallot + IAO & 1 \\
\hline Tetralogía de Fallot + IPulm & 2 \\
\hline Atresia pulmonar + CIV + MAPCAS & 1 \\
\hline VU + RVPAT & 1 \\
\hline Atresia tricuspídea + Estenosis pulm & 1 \\
\hline Ebstein & 1 \\
\hline
\end{tabular}

CIA: comunicación interauricular; CIV: comunicación interventricular; RVPAP: retorno venoso pulmonar anómalo parcial; CAVC: canal aurículo ventricular completo; CAVT: canal aurículo ventricular intermedio; CAVP: canal aurículo ventricular parcial; EMV: estenosis medio ventricular; IAO: insuficiencia aórtica; VU: ventrículo único; TGA: transposición de grandes arterias; RVPAT supra: retorno venoso pulmonar anómalo total supradiafragmático; RVPAT intra: intracardíaco; RVPAT infra: infradiafragmático; DTSVD: doble tracto de salida de ventrículo derecho; EAO: estenosis aórtica; Ipulm: insuficiencia pulmonar.

\section{Tabla 2. Complicaciones}

\begin{tabular}{ll}
\hline Complicaciones & $\mathbf{n}$ \\
\hline Falla cardíaca & $1^{*}$ \\
Hipertensión arterial pulmonar & $4{ }^{*}(2)^{*}$ \\
Bajo gasto & $2^{*}{ }^{*}$ fallecidos \\
Falla multiorgánica & $2^{*}{ }^{*}$ \\
Sepsis & $4^{*}$ \\
Hipoxia + insuficiencia respiratoria & 2 \\
Síndrome postpericardiotomía & 2 \\
Arritmia & 4 \\
Mediastinitis & 2 \\
Parálisis frénica & 1 \\
Sangrado & 2 \\
Obstrucción shunt sistémico pulmonar & 2 \\
Bloqueo aurículoventricular completo & 1 \\
Respiratorias & 1 \\
Urológicas & \\
\hline
\end{tabular}

caso doble salida arterial con CIA restrictiva y los dos restantes retorno venoso pulmonar anómalo total supradiafragmático, en un caso, e infradiafragmático en el otro con coartación de aorta.

Un paciente con tríada de Shone con estenosis aórtica crítica, válvula mitral en el límite inferior normal, que requirió valvulotomía y reparación de coartación de aorta, un paciente con corrección de truncus arterioso y por último un paciente con atresia pulmonar a septum íntegro con sinusoides y ventrículo derecho hipoplásico con Valor Z-4.

Los restantes cuatro pacientes fallecidos antes del alta eran cardiopatías medianamente complejas: tres pacientes con canales atrioventriculares completos (trisomías 21), dos de los cuales tuvieron complicaciones infecciosas y uno respiratoria con estudios ecocardiográficos postoperatorios sin defectos residuales significativos.

Estos pacientes eran portadores de una severa desnutrición por haber presentado insuficiencia cardíaca refractaria al tratamiento médico en el preoperatorio y múltiples infecciones pulmonares que requirieron internaciones prolongadas.

Finalmente, un RN con tetralogía de Fallot y sufrimiento hipóxico isquémico severo perinatal presentó una enterocolitis necrotizante y requirió de un shunt sistémico pulmonar, desarrollando un shock séptico a Gram negativo. 


\section{Discusión}

El resultado final de un procedimiento quirúrgico depende de la interrelación entre la complejidad y la performance del equipo que toma a cargo el paciente.

Sin embargo, cuando se trata del análisis de los resultados del tratamiento quirúrgico de las cardiopatías congénitas, la tarea no resulta para nada sencilla. En esta especialidad la dificultad principal, a diferencia de la cirugía cardíaca del adulto, reside en el hecho de que se debe lidiar con aproximadamente 200 diagnósticos y más de 150 procedimientos ${ }^{11}$, los cuales a su vez combinan otra serie de factores que dependen de cada caso en particular: edad, peso, estado nutricional, síndromes genéticos, estados comórbidos, etcétera.

Las últimas publicaciones ${ }^{11-16}$ coinciden en que la mortalidad al alta hospitalaria podría ser un buen índice para evaluar la performance de una institución.

La mortalidad del 7\% hallada en este análisis se encuentra dentro de los rangos referidos en publicaciones internacionales.

En el estado de New York para el período 1991-1995 en una publicación del año $2001^{12}$ la mortalidad hallada fue de 6,7\%; para el Reino Unido (1997-1999) esa misma publicación refiere una mortalidad promedio para 12 centros de $4,4 \%$, pero con extremos que fueron de $1,8 \%$ a $7,5 \%$. Para Suecia, en el período 1995 1997, fue de 1,9\%. La sociedad de Cirujanos Torácicos de EE.UU. (16 mejores centros) refiere para el período $1998-2001^{13}$ una mortalidad intrahospitalaria que varió entre 3,7\% y $5,6 \%$ y para el año $2002^{14}$ de 2,7 a $7,4 \%$ (promedio 4,2\%).

Uno de los hallazgos que se desprende de este análisis es el importante porcentaje de cirugías correctivas $(87,8 \%)$ en relación con los procedimientos paliativos (9,8\%), lo cual está en acuerdo con las publicaciones actuales ${ }^{1,2,6,8-10,16,17}$. Ello se debe a los avances logrados en el manejo peri y postoperatorio, lo cual ha permitido reducir la edad y el peso en el cual se corrigen las cardiopatías, reservando las cirugías paliativas solamente para casos puntuales.

Con referencia a la complejidad de las cardiopatías intervenidas, aproximadamente la mitad
(48\%) fueron simples y la otra mitad (52\%) complejas o medianamente complejas, reflejando una cierta homogeneidad en la distribución.

El 93\% $(n=14)$ de los decesos correspondió al grupo de pacientes menores de tres meses y $73 \%(n=11)$ eran cardiopatías complejas, reflejando la gravedad de la malformación cuando requiere de un procedimiento quirúrgico en las primeras etapas de la vida. Por su parte, los cuatro pacientes que pertenecían al grupo con cardiopatías medianamente complejas asociaban factores comórbidos que aumentaron significativamente el riesgo quirúrgico (desnutrición, síndrome cromosómico, patología pulmonar).

El estudio RACHS- $1^{11}$, que pretende realizar un ajuste de riesgo, lo hace considerando únicamente el tipo de procedimiento quirúrgico empleado. Más arriba ya hemos enfatizado que son múltiples las variables que intervienen para condicionar el resultado final, sin embargo, debido a la complejidad que significaría tomar en cuenta todos los factores que intervienen para ajustar el riesgo de un determinado procedimiento, nos encontramos con pocas publicaciones ${ }^{11-15,19}$ que nos permitan realizar estudios comparativos, y la mayoría sólo toma en cuenta algunos de ellos. Los que tratan de considerar un número mayor, resultan de tal complejidad que dificultan su aplicación ${ }^{19,21}$.

El RACHS- ${ }^{11}$ diferencia seis categorías de complejidad quirúrgica. La tabla 3 muestra el estudio comparativo entre dicho estudio y los resultados del Centro Cardiológico Americano. Se podrá apreciar que solamente $27,5 \%$ de las cirugías analizadas en nuestra serie están en la categoría 1 (procedimientos "simples"), mientras que las categorías 2-3 (procedimientos "medianamente Complejos" y "complejos") representaron $63,5 \%$ de los procedimientos efectuados.

Evidentemente la diferencia entre ambas muestras (el estudio Rachs ${ }^{1}$ incluye más de 3 mil cirugías) no permite sacar conclusiones desde el punto de vista estadístico. Tomando en cuenta si los procedimientos fueron realizados con o sin $\mathrm{CEC}^{12}$, la mortalidad intrahospitalaria para el Reino Unido varió entre 3,3\% y 7,9\% para las cirugías con CEC y entre $0,8 \%$ y 3,4\% para las cirugías cerradas. 
La hallada en nuestro estudio fue del 8,7\% y 4\% (según hayan sido con o sin CEC).

Otra manera de analizar la mortalidad es según el grupo etario considerado. Así la sociedad de cirujanos torácicos de EE.UU. ${ }^{13}$ refiere $11,2 \%$ para el grupo de 0 a 28 días, 3,2\% para los de 29 días a un año y 1,6\% para los mayores de un año. Los valores hallados por nosotros en este análisis fueron de 15,7\%, 7,2\% y 0\% respectivamente.

Finalmente, en la tabla 4 el estudio comparativo se realiza con el Registro Español de cirugía cardiovascular ${ }^{15}$ según tipo de cirugía y grupo etario, tomando dicho estudio los años 2001, 2002 y 2003.
Cuando se realizan estudios comparativos de mortalidad, y sobre todo cuando se quiere efectuar un ajuste de riesgo, se debe ser muy precavido a la hora del análisis ${ }^{16,20}$. En primer lugar porque las publicaciones internacionales a las que hacemos referencia toman en cuenta a los mejores centros de países desarrollados.

En consecuencia, los resultados van a estar influenciados por otros factores además de los ya considerados: diferencias socioeconómicas, diversidad geográfica de la población, organización de la salud y status global de la atención médica de la región que son inevitablemente el reflejo de la realidad geopolítica donde se ubica el centro.

Tabla 3. Estudio comparativo de mortalidad instrahospitalaria según complejidad quirúrgica. Estudio RACHS-1. J Thorac Cardiovasc Surg 2002; 123: 110-8

\begin{tabular}{|c|c|c|c|}
\hline \multirow[b]{2}{*}{ Procedimientos realizados CCA } & \multicolumn{3}{|c|}{ Mortalidad intrahospitalaria } \\
\hline & $\begin{array}{c}\text { Unidad Card. Cong. } \\
\text { CCA } \\
\%\end{array}$ & (n) & $\begin{array}{c}\text { RACHS-1 } \\
32 \text { centros EE.UU. } \\
\%\end{array}$ \\
\hline $\begin{array}{l}\text { Categoría } 1 \\
\text { Procedimientos "simples" } \\
\mathrm{n}=58(27,5 \%)\end{array}$ & 0 & $(0)$ & 0,4 \\
\hline $\begin{array}{l}\text { Categoría } 2 \\
\text { Procedimientos "med. complejidad" } \\
\mathrm{n}=87(40,8 \%)\end{array}$ & 1,1 & (1) & 3,8 \\
\hline $\begin{array}{l}\text { Categoría } 3 \\
\text { Procedimientos "complejos" } \\
\mathrm{n}=49(23 \%)\end{array}$ & 12,2 & (6) & 8,5 \\
\hline $\begin{array}{l}\text { Categoría 4, 5, } 6 \\
\text { Procedimientos "alta complejidad" } \\
\mathrm{n}=19(9 \%)\end{array}$ & 42,1 & (8) & $\begin{array}{c}19,4-47,7 \\
(\equiv 33,5)\end{array}$ \\
\hline
\end{tabular}

* Se unificaron las categorías 4-5-6 (alta complejidad) por el bajo (n) en c/u. CCA: Centro Cardiológico Americano.

Tabla 4. Estudio comparativo de mortalidad instrahospitalaria según tipo de cirugía y grupo etario. Registro Español de Cirugía Cardiovascular (www.seccv.es)

\begin{tabular}{|c|c|c|c|c|}
\hline \multicolumn{5}{|c|}{ Mortalidad intrahospitalaria } \\
\hline Año & 01 & & 03 & $\begin{array}{c}\text { CCA } \\
03-06 *\end{array}$ \\
\hline & $\%$ & $\%$ & $\%$ & $\%$ \\
\hline Cirugía C/CEC & 4,9 & 6 & 6 & 8,7 \\
\hline C/CEC $<1$ año & 9,5 & 10 & 11 & 14,4 \\
\hline C/CEC > 1 año & 2,7 & 3,7 & 3 & 0 \\
\hline Cirugía S/CEC & 3,5 & 2,3 & 2,3 & 4 \\
\hline
\end{tabular}

C/CEC: con circulación extracorpórea; S/CEC: sin circulación extracorpórea; CCA: Centro Cardiológico Americano. *marzo 2006 
Lamentablemente, no contamos con estudios regionales de países en vías de desarrollo, lo cual permitiría comparar muestras más homogéneas y extraer conclusiones más reales.

También estamos conscientes de que la medicina moderna exige contar con métodos que permitan evaluar y comparar resultados con el fin de informar al paciente y su familia sobre el riesgo potencial de un procedimiento. Sin embargo, como venimos de ver, comparar el riesgo de una determinada institución en lo que refiere al tratamiento quirúrgico de las cardiopatías congénitas resulta dificultoso por la gran cantidad de variables que intervienen ${ }^{16}$.

En segundo lugar, cuando afinamos el análisis y tomamos subgrupos (por ejemplo: edades, con o sin CEC, etcétera) en el caso de la población analizada en este estudio, la muestra disminuye significativamente quedando reducida a decenas de pacientes, en relación a la de los estudios comparativos que toman miles. ¿Cuál es la consecuencia directa? Cada una de las variables constituye un elemento independiente asociado a un riesgo determinado, que dificulta la predictibilidad del resultado final. Su impacto dependerá del tamaño de la muestra y del período de tiempo considerado. Si la muestra es muy pequeña, los factores de riesgo poco frecuentes podrían influir tanto en el resultado final, que su impacto podría ser más importante que la performance real del equipo. Esto, en un sentido como en otro, dado que como vimos para algún subgrupo el resultado hallado fue más favorable que en los estudios comparativos. Cada factor de riesgo en lugar de "diluirse" en una muestra mayor, pasa a adquirir una preponderancia tal sobre el resultado que altera cualquier análisis sobre la performance quirúrgica.

El azar puede hacer que los casos con mayor riesgo operatorio o viceversa se concentren si el número de la muestra es bajo o el período de tiempo considerado escaso ${ }^{16}$.

Lo que estaríamos haciendo sería extrapolar el riesgo individual de un pequeño grupo de pacientes con determinados factores comorbidos y de complejidad (cardiopatía y procedimiento) a un grupo que de ser mayor, hubiera tenido las características de la distribución habitual.

Esto se pone de manifiesto cuando analiza- mos la mortalidad global intrahospitalaria. La hallada de $7 \%$ se compara favorablemente con los referidos en la mayoría de los estudios considerados. Las diferencias comienzan a ser algo mayores a medida que se reduce la muestra para analizar subgrupos, por las razones antes expuestas.

\section{Conclusiones}

A pesar de la realidad que nos separa de los mejores centros de países desarrollados, el resultado del tratamiento quirúrgico por cardiopatía congénita hallado en este estudio se encuentra dentro de los límites referidos en muchos de los estudios comparativos cuando se toma la mortalidad total intrahospitalaria. Las diferencias se acentúan en algunos casos, cuando se consideran subgrupos al reducirse el número de la muestra, no permitiendo sacar conclusiones definitivas.

Existe una marcada tendencia en favorecer la cirugía correctiva de primera intención reservando la paliativa para casos puntuales (elevado riesgo, contraindicación jemodinámica).

Los decesos fueron en pacientes con cardiopatías complejas o medianamente complejas con factores comórbidos que aumentaban significativamente el riesgo quirúrgico.

La mayor mortalidad la posee el grupo de menores de un año y dentro de él el de los recién nacidos, reflejando la gravedad de las cardiopatías que requieren tratamiento quirúrgico en etapas tempranas de la vida.

\section{Referencias}

1.- Castañeda A: Congenital Heart Disease: A surgicalHistorical perspective. Ann Thorac Surg 2005; 79: S 2217-20.

2.- Freedom RL, Lock J, Bricker JT: Pediatric Cardiology and cardiovascular Surgery: 1950-2000. Circulation 2000; 120: IV-58.

3.- Gott VL: Lillehei, Lewis and Wangensteen: The right mix for giant achievements in cardiac surgery. Ann Thorac Surg 2005; 79 (6): S2210-3.

4.- Reddy VM, Mcelhinney DB, Sagrado T, Parry AJ, Teitel DF, Hanley FL: Results of 102 cases of complete repair of congenital heart defects in patients weighing 700 to 2500 grams. J Thorac Cardiovasc Surg 1999; 117: 324-31. 
5.- Chang AC, Hanley FL, Lock JE, Castañeda AR, Wessel DL: Management and outcome of low bith weight neonates with congenital heart disease. J Pediatr 1994; 124: 461-6.

6.- Van Arsdell GS, Maharaj GS, Tom J, Rao VK, Coles JG, Freedom RM, et al: What is the optimal age for repair of tetralogy of Fallot? Circulation 2000; 102: 11123.

7.- Choudhary SK, Talwar S, Airan B, Mohapatra R, Juneja $R$, Kothari SS, et al: A new technique of percutaneously adjustable pulmonary artery banding. J Thorac Cardiovasc Surg 2006; 131: 621-4.

8.- Merrick A F, Lal M, Anderson RH, Shore DF: Management of ventricular septal defects: A survey of practice in the United Kingdom. Ann Thorac Surg 1999; 68: 983-8.

9.- Bhatt M, Roth SJ, Kumar RK, Gauvreau K, Nair SG, Chengode S, et al: Management of infants with large, unrepaired ventricular septal defects and respiratory infection requiring mechanical ventilation. J Thorac Cardiovasc Surg 2004; 127: 1466-73.

10.- Albus RA, Trusler GA, Izukawa T, Williams WG: Pulmonary artery banding. J Thorac Cardiovasc Surg 1984; 88: 645-53.

11.- Jenkins KJ, Gauvreau K, Newburger JW, Spray TL, Moller JH, Iezzoni LI: Consensus based method for risk adjustment for surgery for congenital heart disease. J Thorac Cardiovasc Surg 2002; 123: 110-8.

12.- Stark JF, Gallivan S, Davis K, Hamilton JR, Monro JL, Pollock JC, et al: Assessment of mortality rates for congenital heart defects and surgeons' performance. Ann Thorac Surg 2001; 72: 169-75.

13.- Jacobs JP, Mavroudis C, Jacobs ML, Lacour-Gayet FG, Tchervenkov CI, Gaynor JW, et al: Lessons learned from the data analysis of the second harvest (19982001) of the Society of Thoracic Surgeons (STS) Congenital Heart Surgery Database. Eur J Cardiothorac Surg 2004; 26: 18-37.

14.- Jacobs JP, Lacour-Gayet FG, Jacobs ML, Clarke DR, Tchervenkov CI, Gaynor JW, et al: Initial Application in the STS Congenital Database of Complexity Adjustment to evaluate Surgical Case Mix and Results. Ann Thorac Surg 2005; 79: 1635-49.

15.- Sociedad Española de Cirugía Torácica y Cardiovascular: Registro Español de Cirugía Cardiovascular [en línea]. Madrid: SECTCV, 2004 <www.seccv.es> [consulta: 20 jul 2006].

16.- Williams W: Defining operative mortality: It should be easy, but is it? Ann Thorac Surg 2006; 81: 155760.

17.- Prifti E, Bonacchi M, Bernabei M, Crucean A, Murzi B, Bartolozzi F, et al: Repair of complete atrioventricular septal defects in patients weighing less than $5 \mathrm{~kg}$. Ann Thorac Surg 200; 1717-26.

18.- Bando K, Turrentine MW, Sun K, Sharp TG, Ensing GJ, Miller AP, et al: Surgical management of complete atrioventricular septal defects a twenty-year experience. J Thorac Cardiovasc Surg 1995; 110: 1543-54.

19.- Lacour-Gayet F: Quality evaluaiton in congenital heart surgery. Eur J Cardiothorac Surg 2004; 26: 1-2.

20.- Ribeiro ALP, Gagliardi SPL, Nogueira JLS, Silveira LM, Colosimo EA, Lopes do Nascimento CA: Mortality related to cardiac surgery in Brazil 20002003. J Thorac Cardiovasc Surg 2006; 131: 907-9.

21.- Kang N, Tsang VT, Elliott MJ, de Leval MR, Cole TJ: Does the Aristotle Score predict outcome in congenital heart surgery? Eur J Cardiothorac Surg 2006; 29: 98690 . 